\title{
Consecuencias de la incorporación de España a la Europa verde
}

\author{
Julián Alonso Fernández
}

\begin{abstract}
El dedicar una sesión exclusiva al tema de la Agricultura requiere de algunas explicaciones: La Agricultura, como actividad económica, presenta características particulares que la diferencian de otras actividades tales como la Industria o los Servicios. Entre ellas destacan: a) la actividad agraria conlleva un modo de vida rural y unas específicas condiciones de trabajo, b) la producción agraria tiene que cubrir las necesidades alimenticias de la población, con un sistema de oferta-demanda rígido, puesto que la demanda se va produciendo a lo largo de todo el año y la cosecha (oferta) sólo en una época determinada, $c$ ) el proceso de producción está condicionado por factores aleatorios, tales como la climatología, las características naturales de la tierra, etc.
\end{abstract}

Precisamente por estas características los diferentes Estados se ven motivados a diseñar políticas agrarias que garanticen una renta adecuada a los productores y una oferta a los consumidores, y a precios razonables.

La diversidad de producciones y de necesidades de consumo de los primeros seis países miembros era enorme, presentando tanto elementos de complementariedad como de solapamiento, lo que exigia gran trabajo para armonizar las políticas agrarias propias de cada uno de ellos.

Los seis países firmantes del Tratado cubrían tan sólo el 85 por 100 de sus necesidades alimenticias y presentaban un número elevado de población activa agraria. Así, los objetivos a conseguir por la Politica Agraria Común (PAC) se centraban en un crecimiento de las producciones que garantizasen el autoabastecimiento, y en una reducción de la población activa agraria. 
Hoy la PAC es la más importante de las políticas comunitarias, absorbiendo en torno al 70 por 100 del presupuesto de la CEE.

El Mercado Común Agrícola se funda en tres principios básicos: a) la unidad de mercado; $b$ ) la preferencia comunitaria; $c$ ) la solidaridad financiera.

La Europa verde, lo que debiera ser el Mercado Común Agricola, viene configurada por la Política Agraria Común. A pesar de tener que ser remodelada constantemente es una de las políticas comunitarias más consolidadas. Ya ha conseguido sobradamente su objetivo de dar de comer a los ciudadanos europeos y de garantizar a los agricultores un alto nivel de vida. La PAC tiene de positivo la seguridad del aprovisionamiento en alimentos, la modernización técnica y productiva, los beneficios económicos y la posibilidad de comerciar internacionalmente; y de negativo el que se hace a cuenta de unos costos enormes, de unas fuertes succiones del presupuesto, de que realmente no regula los mercados ni se adapta bien a las demandas de los consumidores.

La PAC se fundamenta en cinco objetivos: a) elevar la productividad del sector; b) asegurar a los agricultores un nivel de vida similar al de otros trabajadores; $c$ ) estabilizar los mercados; $d$ ) conseguir la continuidad y regularidad en los aprovisionamientos; e) conseguir los aprovisionamientos a precios razonables.

Para conseguirlo, lo adecuado es buscar la complementariedad de las regiones, pero en ningún momento se ha modificado en nada la estructura previa.

En 1960 se formularon las líneas que vertebrarian la PAC, que se concretaban: a) en una política de mercados y precios; $b$ ) una política comercial para la relación con terceros; $c$ ) una política de modernización de las estructuras agrarias, y $d$ ) una política social.

Esta se estructuró mediante determinadas medidas en favor de los agricultores, contemplando, entre otras, la de un programa de formación (agraria y empresarial) y una estrategia de jubilaciones para modernizar el campo en su factor decisivo: el agricultor; medidas de mejora de las explotaciones, acudiendo a su redimensionamiento o adecuación técnica. Asimismo se acude a medidas en favor de la comercialización y de la transformación de productos. 
La PAC quizá sea víctima de su propio éxito, pues, al mejorar sustancialmente la agricultura, ha generado excedentes difíciles de colocar en el mercado interior, donde la demanda está saturada, y casi imposible de transferir a otros mercados, donde no hay demanda solvente capaz de adquirirlos: al ser los costos de producción más altos, son, por consiguiente, más altos los precios.

La Comunidad ha desarrollado una verdadera política europea en el terreno agrícola. Es, prácticamente, la única política comunitaria: a) las organizaciones de mercado han permitido la defensa y la estabilización de las rentas agrícolas. Los precios, más altos siempre que los nacionales anteriores, han ocasionado un aumento de los ingresos agricolas; $b$ ) la mejora de la productividad agrícola, primer objetivo que el Tratado de Roma asignó a la PAC, es indiscutible; c) la localización geográfica de la actividad agrícola da lugar a un enfrentamiento de tendencias: de una parte, el deseo de provocar en Europa una localización óptima de la producción agrícola, la que asegura un cierto volumen de producción a un coste mínimo (es el problema de la especialización) y, de otra parte, el deseo de no ver desaparecer la agricultura de ciertas regiones en donde contribuye a la actividad económica (es el problema de la ayuda a los agricultores peor situados). En estos aspectos apenas se ha conseguido nada.

Para la agricultura española, la adhesión a la CEE supone un reto de adaptación a un mercado mucho más competitivo, ya en esta situación actual. Proceso necesario y que permitirá beneficiarse del acceso a una zona económicamente más amplia, basada en la libertad de mercado, sin barreras aduaneras y con preferencia comunitaria de nuestros productos frente a países terceros.

Está claro que la total integración de España en la CEE ofrece un cúmulo de facetas que condicionará marcadamente el futuro de nuestra agricultura. Pero si tenemos en cuenta que la PAC se programa y se readapta cada año, deduciremos lo dificil que es determinar este nuestro futuro agrario. Salta a la vista, sin embargo, que desde el punto de vista de los agricultores y considerando el problema globalmente, se obtendrán más beneficios que daños, una vez haya transcurrido el periodo transitorio: hay que tener presente que la Comunidad Económica Europea ofrece unos niveles de protección y de ayuda a la agricultura muy superiores a los que se vienen aplicando en España. Actualmente en nuestro país predominan las pequeñas explotaciones, la parcelación es excesiva, las estructuras comerciales son inadecuadas por lo que no benefician a las rentas del campesino, tienen escaso peso las agrupaciones de productores agrarios capaces de comercializar, etc. 
La filosofía que impregna al Mercado Común Agrícola se apoya en la teoría de los grandes mercados, o sea, espacios territoriales amplios en cuyo seno es posible la división del trabajo y los intercambios libres, sin limitaciones de tipo geográfico, aduanero, monetario, administrativo, etc. en un marco, por consiguiente, de libre competencia absoluta. Ello se traduce, por un lado, en la especialización de las empresas y en su agrandamiento para hacerse más competitivas $\mathrm{y}$, por otro, en seguridad de aprovisionamientos regulares y en estabilidad en la relación producción/venta. Pero en el Mercado Común se presenta el problema de que las producciones de los diversos Estados son complementarias en unos casos, mientras que en otros son concurrentes. En el primer supuesto no hay incentivos al progreso, ni problemas; en el segundo, la evolución se hace imprescindible.

En resumidas cuentas, en teoría de economía general, este gran mercado agrario europeo favorece la localización de la producción de forma más ventajosa y en empresas más avanzadas. La agricultura, directísimamente ligada al espacio y a las específicas características de éste, parece la actividad por excelencia para la creación de un vasto mercado, que ha de producir nuevas localizaciones de los productos teniendo en cuenta la fertilidad de las diversas áreas y las localizaciones de los centros de consumo. En consecuencia, algunos productos que tenían mercado en una economía nacional, no lo tendrán en una supranacional y habrán de considerarse marginales. Las regiones con más condiciones favorables para estos productos los obtendrán a más bajos precios, aumentarán las cantidades y arruinarán las de los sectores con peores condiciones para su obtención.

Sin embargo, en la práctica, este liberalismo económico absoluto, este juego simple de las leyes del mercado, no se aplica. No se aplica porque va contra el objetivo de la agricultura comunitaria de garantizar, a toda costa, el aprovisionamiento alimenticio para todos los habitantes, no carecer de alimentos $y$, además, a precios sin oscilaciones distorsionadoras, aun cuando éstos sean más altos que en el mercado mundial; porque el objetivo es elevar el nivel de vida de los agricultores y aproximarlo al del resto de los sectores. Para ello Europa ha potenciado un elevadísimo grado de mecanización, una intensiva utilización de fertilizantes que, en productos concurrentes, ha llevado a la aparición del problema de los excedentes. El exceso de producción es un problema básico, puesto que los excedentes son financiados a muy alto costo y han obligado a reglamentaciones, producto a producto, muy minuciosas.

La PAC, en constante retoque, se basa, como he reiterado y es bien sabido, en: a) el libre tráfico de productos agrarios por todos los países de 
la Comunidad; $b$ ) la protección aduanera frente a países extracomunitarios; c) la solidaridad financiera (FEOGA) para: la ordenación del mercado y la orientación de la producción, con el objetivo de sostener los ingresos de los productores agrícolas; para la transformación de las estructuras y para las atenciones sociales a los campesinos. La ordenación común del mercado de los diversos productos se apoya fundamentalmente en la política de precios.

El ingreso de España, esto es fundamental, nos obliga a homologar nuestra legislación y reglamentos de mercados, precios, subvenciones, asistencia, etc. con la europea, lo que en mi opinión, es muy positivo para nuestro agro, por cuanto: 1) supone una sustancial mejora de la asistencia social (sanitaria, paro, pensiones, accidentes) en relación a la situación española actual. En la Comunidad está establecido el CEPFAR: Centro Europeo para promoción y formación en el medio agrícola y rural; 2) garantiza mejor las posibilidades de trabajo, al favorecer la movilidad de personas por toda Europa, sin obstáculo ni discriminación alguna; 3) apoya especialmente la agricultura de montaña, promueve mejoras en las áreas deprimidas, incentiva la jubilación de los campesinos de edad; 4) financia la reforma de las estructuras con el objeto de lograr unas explotaciones de tamaño y condiciones socioeconómicas más racionales, aliviando el problema, social y económico, de los latifundios y minifundios. Para ordenar las producciones, precios, etc. el Mercado Común invierte mucho más dinero que España, más del doble para la mejora de estructuras y de redes comerciales, e incluso más. Aquí sin duda está el germen de una nueva agricultura y de una reforma agraria eficaz; 5) proporciona información socioeconómica al productor y cuida de su cualificación profesional. Además promueve las agrupaciones y confederaciones de productores, obligatorias en la Comunidad: COPA: Comité de organizaciones profesionales agrarias. CEJA: (dentro del COPA): Consejo europeo de Jóvenes Agricultores. EFA: Federación europea de trabajadores agrícolas. COGECA: Comité General de la Cooperación Agricola. No obstante, son objetivos a aplicar gradualmente. Se comprueba que, a pesar de lo que dije antes sobre los efectos de la aplicación de la teoría de los grandes mercados, desde que existe la PAC, en nada se ha modificado la localización nacional y regional de los productos agrícolas; si bien es verdad que, por otro lado, las transacciones intracomunitarias de bienes agrarios se han incrementado espectacularmente.

No son de prever cambios radicales en las características actuales de nuestro sector agropecuario, porque a la integración absoluta en el Mercado Común Europeo precede un periodo transitorio, durante el que las consecuencias de la adhesión van produciéndose poco a poco. Las medidas del 
periodo transitorio, en cuanto a la Organización Común de Mercados, obligan a unas aproximaciones progresivas a los precios comunitarios, mediante mecanismos muy variados. Por todo ello, las indudables ventajas de conjunto de la incorporación, sólo surtirán efecto a largo plazo, al estar mermadas durante el período transitorio. Por ejemplo, los agrios se encontrarán con situaciones bastante más penosas que las que tienen terceros paises mediterráneos, especialmente Marruecos e Israel, por aplicación de fuertes derechos aduaneros. Sin duda, la lentitud tiene algo de positivo: así, una equiparación demasiado rápida de los precios y de todas las normas desequilibraría los mercados. Por ejemplo, en el subsector pecuario, la subida rápida del precio de los piensos y forrajes afectaría gravemente a la rentabilidad de las granjas españolas, y a los consumidores; la reducción del precio de la leche, obtenida, sobre todo, en pequeñas granjas familiares, arruinaría a nuestros ganaderos. A las ventajas que puede obtener España en los productos más competitivos, los mediterráneos, hay que oponer la grave desventaja en típicos productos europeos (bovino, leche y derivados, etc.) que, protegidos con altos precios en España para favorecer antaño su desarrollo, habrán de ser reducidos para poder competir, o habrán de ser reordenados. En general, la competencia será difícil con nuestros climas y suelos, nuestra baja productividad por $\mathrm{Ha}$. y nuestras deficientes estructuras productivas (referidas a tamaño de las explotaciones, descapitalización, escaso nivel tecnológico, población envejecida, etc.). Los productos mediterráneos parecen los protagonistas y esto nos hace olvidar que la agricultura mediterránea supone menos del 40 por 100 del total, que también tenemos una agricultura atlántica en el norte y noroeste, una agricultura de montaña y una agricultura continental, muy débil, en la Meseta, que no tienen demasiado favorables perspectivas.

La Europa Norte absorbe la mayor parte de los gastos del FEOGA, en detrimento de la agricultura mediterránea, que además presenta importantes problemas socioestructurales. Este debate permanente mantenido en el seno de la Comunidad va a contar a partir de ahora con un elemento más de discusión: el crecimiento de la agricultura mediterránea en la CEE con la integración de España y Portugal.

La agricultura mediterránea de los diez habia alcanzado ya en 1984 un buen nivel de autoaprovisionamiento en líneas generales, aunque en determinados productos aparece como claramente excedentaria y, en otros, por el contrario, como deficitaria o muy deficitaria. En cuanto a los primeros, aquellos en los que los excedentes son muy importantes, los productos más señalados son el trigo, la cebada, el azúcar, vino, aceite y huevos. Estos productos, con más elevados costes de producción que en otros lugares, no 
tienen salida a los mercados exteriores, a menos que se ofrezcan por debajo de su precio de coste. La situación contraria se origina con otra serie de productos en los que la Comunidad es claramente deficitaria como, por ejemplo, el maíz, el tabaco, los agrios, el algodón, la fruta, el arroz y algunas hortalizas. A éstos se añaden diversas grasas vegetales que, como la soja, el cártamo, el girasol, etc. son básicas para la elaboración de piensos, tan importantes en una economía en la que los productos ganaderos representan más del 50 por 100 del valor final de la producción agraria. Un nivel equilibrado apenas se da en otros productos que en la patata, leche fresca, carne de bovino y de cerdo.

Pero, con la entrada de España y de Portugal esta situación se alteró: en algunos casos empeoró por aumentar con ello los excedentes, como sucedió con el vino y el aceite; en otros empeoró por lo contrario, por hacer mayor el déficit, caso del maíz. Pero en muchos otros aspectos la situación ha mejorado, bien por reducir excedentes estructurales de la anterior CEE al ser los países ibéricos deficitarios (cebada y azúcar, por ejemplo), bien por contribuir a acercarse al nivel de autoabastecimiento (casos de la fruta, agrios, arroz, hortalizas y algodón).

¿Consecuencias?: A futuro creo que se producirá un incremento de las extensiones destinadas a cultivos tales como los de trigo duro, cereales pienso, girasol, soja, y una reducción de las ocupadas por trigo blando (para cebada, girasol o trigo duro), maíz, remolacha azucarera, pero sin olvidar lo difícil que va a ser reconvertir el maíz y la remolacha de los regadíos del Duero y del Ebro. En el subsector ganadero cabe esperar una disminución en la obtención de leche y un moderado incremento en carne de ovino. Será diticil reconvertir la actual dedicación ganadera en Galicia, Asturias y Cantabria, por lo que es imprescindible racionalizar las unidades de explotación, si se quieren evitar graves consecuencias a nivel regional.

La Geografia de la Agricultura no cambiará mucho. De hecho esto es lo que ha ocurrido en los territorios de los anteriores diez miembros.

En general, los efectos pueden ser positivos, sobre todo una vez reformadas las estructuras. De lo contrario, los sectores cerealistas, remolachero, cárnico y lechero, que suponen el 40 por 100 de la total producción final agraria, conocerán desagradables efectos. Las exportaciones, más cuantiosas y mejor pagadas, serán el más positivo efecto de la integración.

El ingreso en el Mercado Común ha sido el logro menos desfavorable desde el punto de vista de la actividad agraria. No debemos olvidar que el 
Mercado Común Agricola es el espacio mercantil y productivo con los más elevados precios a la producción y al consumo, lo que acarrea mayores beneficios a las exportaciones de nuestros productos. España comerciaba ya, sobre todo, con la Comunidad Económica Europea y, por lo tanto, suprimidos los derechos aduaneros, mejorará la situación al beneficiarse de la preferencia comunitaria, de la tarifa aduanera común frente a otros paises mediterráneos con hasta ahora acuerdos preferenciales más ventajosos. 